\title{
Toward Effective Needle Steering in Brain Tissue
}

\author{
J.A. Engh ${ }^{1,2}$, G. Podnar ${ }^{1}$, D. Kondziolka ${ }^{2}$, and C.N. Riviere ${ }^{1}$ \\ ${ }^{1}$ The Robotics Institute, Carnegie Mellon University \\ ${ }^{2}$ Department of Neurological Surgery, University of Pittsburgh \\ Pittsburgh, PA 15213
}

\begin{abstract}
Recent research has exploited the inherent bending of a bevel-tipped needle during insertion, accomplishing steering of the needle by rotating the needle shaft. Combining this technique with the observation that a straight trajectory can be accomplished by spinning the needle at a constant rate during insertion, this paper presents a novel technique for proportional control of the curvature of the trajectory via duty-cycled spinning of the needle. In order to accommodate this technique to very soft tissues such as the brain, several custom needle prototypes have also been designed in order to increase the steering versatility of the system by maximizing the attainable curvature. The paper describes the needle-steering system and the needle prototypes, and presents preliminary results from tests in an artificial brain tissue substitute.
\end{abstract}

\section{INTRODUCTION}

Brain cancer is a devastating disorder, and long-term survivorships are exceedingly rare. More than 13,000 Americans die each year from primary brain tumors, and many thousands more from intracranial metastases [1]. Surgical resection is not curative, and radiation therapy adds only a modest benefit to mean survival times. Because of the diffuse histological nature of these lesions, the creation of injectable systemic therapeutic agents with an affinity for tumor cells is an area of active research. However, none of these systemic therapies has ever demonstrated more than a modest benefit in any randomized trial. This failure is partially due to the selectivity of the blood-brain barrier, which is extraordinarily resistant to the passage of large molecules, including chemotherapeutic agents, labeled toxins, and antibodies. Modulation of blood-brain barrier selectivity to improve delivery of systemic agents to the brain is one possible solution to the problem, but success has not yet been realized [2].

An alternative approach to the problem of delivering therapeutic agents across the blood-brain barrier is compartmental therapy, also known as local tumor therapy. This technique has the advantage of bypassing the bloodbrain barrier for direct delivery of a therapeutic agent. Because primary tumor recurrences tend to be within the zone of a prior surgical resection [3], this strategy directly addresses the most significant threat to life in these patients. The most successful local therapy for primary tumors to date is Gliadel (MGI Pharma Inc., Bloomington, MN), a carmustine-impregnated wafer which is implanted into the

Funding provided by the Simeon M. Jones, Jr. and Katharine Reed Jones Fund and the Walter L. Copeland Fund of The Pittsburgh Foundation, and by the National Science Foundation (grant no. EEC-9731748). bed of a resected tumor prior to wound closure. The limited but quantifiable efficacy of carmustine wafers in the treatment of gliomas has been demonstrated in multiple randomized trials [4-6].

Delivery of local therapeutic agents into a resection bed after craniotomy is not the only effective application technique. Agents can also be delivered stereotactically through a catheter in minimally invasive fashion. In fact, the NIH Brain Tumor Progress Group has emphasized that catheter placement for compartmental therapy is a critical component of future treatments for brain tumors [7]. One example is convection-enhanced delivery, in which therapeutic molecules are infused past the blood-brain barrier via a microcatheter into a defined treatment zone [8]. This approach has the advantage of application of the necessary agent past the blood-brain barrier. Therapeutic molecules are distributed from the application site into the surrounding tissue to some extent. However, the molecular concentration of infused molecules within the delivery zone can vary because of heterogeneity of the tumor or the surrounding brain [9]. The efficacy of this approach might be improved by methods for accurate navigation of the needle or microcatheter within a treatment zone.

Techniques for steering of flexible needles through tissue have been a focus of recent research [10-13]. Some techniques rely intrinsically on significant deformation of the tissue, especially near the point of insertion, making them unsuitable for use in the brain. One technique that does not involve large deformation of tissue is based on the observation that a flexible bevel-tipped needle tends to bend during insertion. Webster et al. have developed a technique that exploits this phenomenon in order to steer the needle by rotating the shaft during insertion to determine the direction of bending $[12,13]$. In the course of adapting this technique for brain tissue, the authors have recently developed a simple means by which the amount of bending (or the curvature) can be controlled proportionally as well [14].

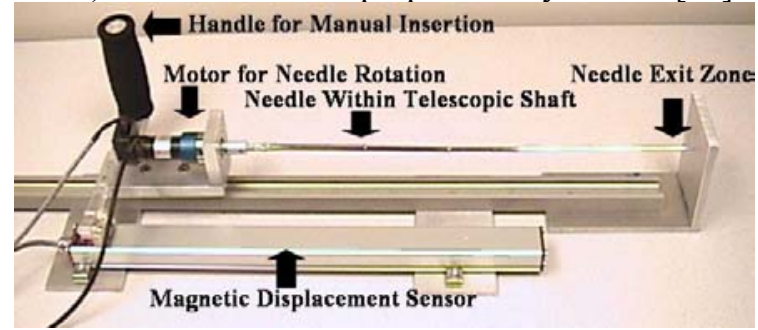

Figure 1: Needle steering prototype. The needle is inserted manually, and the motor is computer-controlled to steer the needle as desired, via dutycycled spinning. The telescopic shaft prevents bending of the uninserted portion of the flexible needle. As the needle is inserted, a magnetic displacement sensor measures the depth of insertion. 
The softness of brain tissue limits the reaction forces during needle insertion, thus limiting the attainable curvature [14]. The authors therefore have developed custom needle prototypes to increase the maximum attainable curvature, thus increasing the versatility of this approach. This paper describes the proportional steering technique and the new needle prototypes, and presents preliminary test results.

\section{METHODS}

\section{A. Design}

The technique presented in [12] provides an elegant means to steer the needle by rotation of the needle shaft during insertion. This technique provides control of the direction of needle curvature, but does not alter the amount of curvature. However, there is a simple technique that can achieve a straight trajectory, namely, spinning the needle at a constant rate during insertion. Given these two facts, arbitrary trajectories in 3-D can easily be envisioned by combining short segments, some straight and some curved, as desired. Taking this to its logical conclusion, any curvature between these two limits (with and without spinning) can be achieved by spinning the needle with a duty cycle, provided the angle of rotation is controlled such that the bevel stops in the same orientation during each "stop" portion of the cycle. Longer "stop" intervals create steeper curvature of the needle, and longer "spin" intervals create a straighter trajectory. The frequency of the duty cycle can easily be set such that the surgeon will perceive the result as a smooth curve rather than a concatenation of straight and curved segments. This technique thus provides proportional control not only of the direction but also the amount of needle curvature.

The first prototype of the needle-steering system is depicted in Fig. 1. Insertion is performed manually. Otherwise the prototype is very similar to that presented in [13]. The electric motor beneath the handle rotates the needle shaft as needed during insertion. The uninserted portion of the needle is kept within a telescoping shaft in order to prevent unwanted bending. A magnetic linear displacement sensor monitors the depth of insertion, in order to provide feedback to the computer that can be used to enable following of a preplanned trajectory, when desired.

Hollow stainless steel needles and solid nitinol wires have been tested with the system. The nitinol wires have yielded superior results, and were therefore chosen for the experiments reported here. The long-term plan is to use hollow nitinol needles. (Nitinol is used here solely because of its elasticity, not because of the shape memory effect.)

The needle curvature depends on the flexibility of the shaft, which can be maximized by decreasing the diameter. However, this involves an unfortunate tradeoff: decreasing the diameter also decreases the surface area of the bevel, which is the bearing surface that presses against the tissue to provide bending. Therefore, two types of custom needles have been investigated in an attempt to increase the maximum attainable curvature. The first type involves applying a kink in the needle shaft, proximal to the beveled tip, thus increasing, in a sense, the effective beveled surface area applied to the tissue. The second type can be thought of as the addition of a sort of "airfoil" to the tip; it involves the addition of an enlarged tip to increase the surface area of the bevel while retaining the narrow gauge of the needle shaft. This can be accomplished by cutting off the tip of a larger needle and attaching it to the end of a narrow-gauge shaft. The effectiveness of this second type can then be further augmented by bending the main shaft to generate a kink proximal to the enlarged tip, as before. Tips as large as 16 gauge have been investigated. Although this is large compared to the main shaft gauge of these custom needles, it is still relatively small compared to various types of needles or cannulae that are routinely used clinically.

\section{B. Testing}

To test needle steering in vitro, the authors used gelatin (Knox gelatin, Kraft Foods Global Inc., Tarrytown, NY) as a tissue substitute. The gelatin was mixed at a ratio of $20 \mathrm{cc}$ boiling water to $1.3 \mathrm{cc}$ gelatin. This mixture has been proposed in the literature as an artificial brain tissue substitute for studies of this type, since the insertion force profile of a small catheter is similar to that of brain tissue in vitro [15]. The gelatin was prepared in a small bowl and a small hole was cut in the side of the bowl for insertion of the needle. A new gelatin sample was used for each test in order to avoid any inhomogeneity caused by preexisting needle tracks that might influence the trajectories of subsequent tests. A mat marked with gridlines was placed at the bottom of the bowl as an aid to visualization.

Two custom needle prototypes were demonstrated. The first was a 34 -gauge nitinol wire $(0.15 \mathrm{~mm}$ diameter $)$ cut at a bevel of $20^{\circ}$ and bent (kinked) to an angle of $16^{\circ}$ at a point $6.3 \mathrm{~mm}$ from the tip. The second consisted of the tip of a 16-gauge (1.27 $\mathrm{mm}$ diameter) stainless steel hypodermic needle with a $10^{\circ}$ bevel, attached to a 29-gauge nitinol wire $\left(0.28 \mathrm{~mm}\right.$ diameter) kinked to a $15^{\circ}$ angle at a point $6.3 \mathrm{~mm}$ from the tip. Each of the two custom needle prototypes was tested by inserting it, without spinning, into a fresh gelatin sample.

Proportional steering control was demonstrated using the second custom needle prototype. In each test, each cycle consisted of one full rotation of the needle at $2 \mathrm{~Hz}$, followed by sufficient time without rotation to produce the desired duty cycle. Tests were conducted at duty cycles of $0 \%$, $33 \%, 67 \%$, and $100 \%$. The needle was steered in the horizontal plane.

In addition to the tests above, simple qualitative demonstrations of two possible treatment scenarios were performed in fresh gelatin samples using simple plastic and metal targets and obstacles. One demonstration involved passing through a small wire wicket after steering around an obstacle. The other test demonstrated the ability to reach the vicinity of a lesion via a single trajectory and then provide "coverage" of the volume of a lesion by reaching multiple points within the lesion zone via local steering maneuvers. The second custom needle prototype was used for both of these tests. 


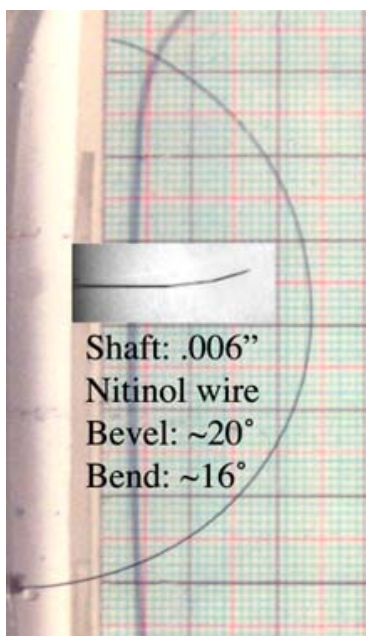

(a)

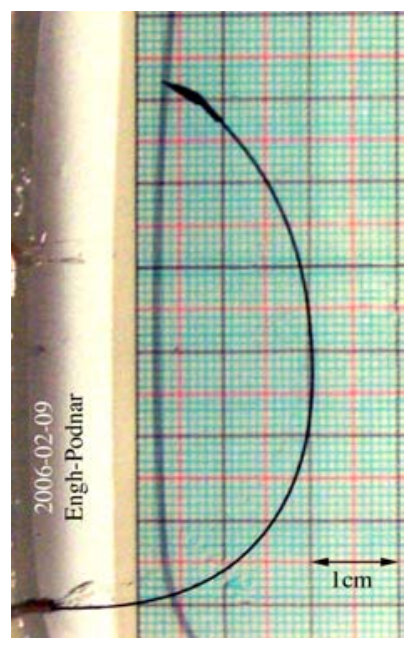

(b)

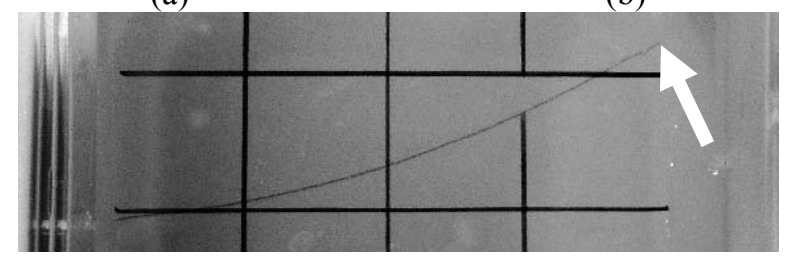

(c)
Fig. 2. Bending of needles, without spinning, in gelatin. The dark gridlines in (a) and (b) are $1 \mathrm{~cm}$ apart; the gridlines in (c) are $2.5 \mathrm{~cm}$ apart. (a) First custom needle prototype: 34 -gauge nitinol wire $(0.15 \mathrm{~mm}$ diameter $)$ cut at a bevel of $20^{\circ}$ and bent (kinked) to an angle of $16^{\circ}$ at a point $6.3 \mathrm{~mm}$ from the tip. (b) Second custom needle prototype: the tip is from a hypodermic stainless steel needle $1.27 \mathrm{~mm}$ in diameter, with a $10^{\circ}$ bevel. The diameter of the main shaft is $0.28 \mathrm{~mm}$ in diameter, and is bent $15^{\circ}$ at a point $6 \mathrm{~mm}$ from the tip. (c) A straight 29 -gauge nitinol wire $(0.28 \mathrm{~mm}$ diameter $)$ with a $45^{\circ}$ bevel (shown for comparison).

\section{RESULTS}

The curvature obtained using the two custom needle prototypes, without spinning, is pictured in Fig. 2. The relatively modest curvature exhibited in this very soft substrate by bevel-tipped straight needles - the result which led to the investigation of custom needle profiles to increase the attainable curvature-is depicted in Fig. 2(c).

The results of the duty-cycled steering tests are presented in Fig. 3. The trajectory generated by continuous spinning (100\% duty cycle) is shown in Fig. 3(a). The maximally curved trajectory achieved with no spinning is shown in Fig. 3(d). The remaining photographs show intermediate amounts of curvature, at duty cycles of $67 \%$ and $33 \%$.

In Fig. 4, a qualitative demonstration is provided of a detour around a plastic bottle cap (representing some critical part of the brain) followed by passage through a small metallic wicket (representing a target lesion). Fig. 5 presents a qualitative demonstration of multi-point "coverage" of a lesion zone. In this example, the needle was steered to the edge of a treatment zone (A), represented by a plastic bottle cap placed within the tissue substrate, with a small hole cut in its side for entry. It was then advanced straight forward to point (B). The needle was then retracted to the entry point (A), and then advanced to other points in the treatment zone $(C$, then $D)$, each time returning to the same starting point (A).

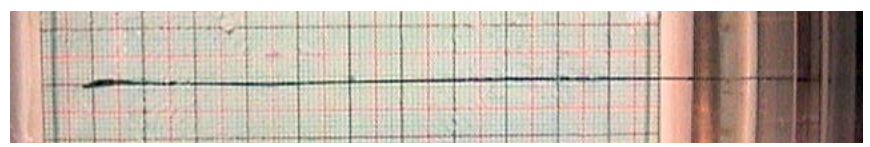

(a)

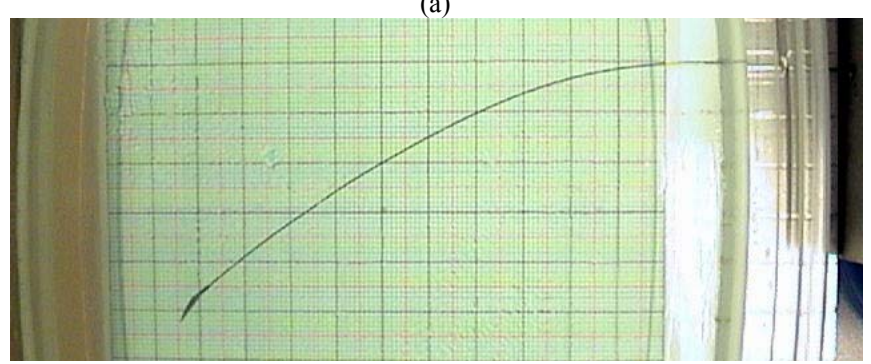

(b)

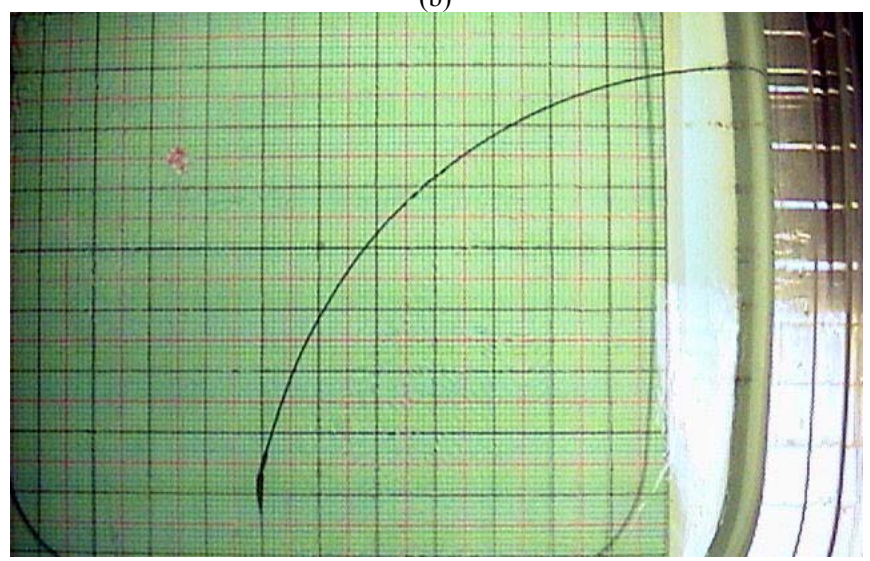

(c)

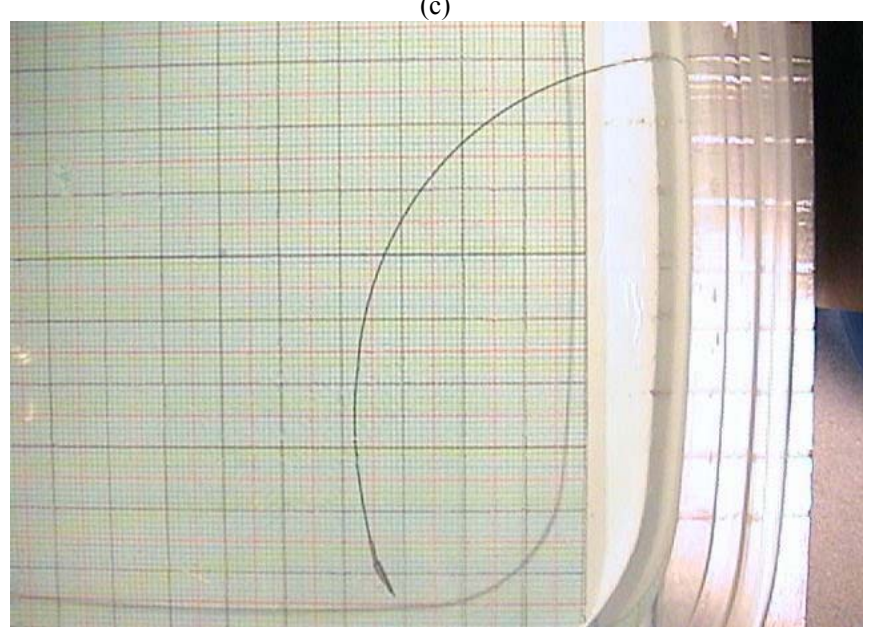

(d)

Fig. 3. Steering of a flexible needle by spinning it at $2 \mathrm{~Hz}$ with various duty cycles during insertion in a gelatin substrate. The needle enters from the right. (a) Continuous spinning (100\% duty cycle). (b) $67 \%$ duty cycle. (c) $33 \%$ duty cycle. (d) No spinning ( $0 \%$ duty cycle). The needle is the second custom prototype needle (Fig. 2(b)). The dark gridlines are $1 \mathrm{~cm}$ apart.

\section{Discussion}

The ability to accurately guide a flexible needle along a relatively arbitrary trajectory within the brain creates a variety of possibilities in the realm of tumor surgery. Trajectories can be planned preoperatively [16], and possibilties for feedback sensing for closed-loop execution of such trajectories include ultrasound [17], magnetic tracking, and medical imaging systems. 
In addition to the possibility of injections through the needle, the use of the needle as a guide wire over which to advance a microcatheter could be used to create a larger working channel for endoscopic tumor resection. Because of the minimally invasive nature of the device, such procedures could be performed on an awake patient through a burr hole craniostomy. Deep-seated eloquent zones could be manipulated while monitoring the patient for any incipient neurologic deficit. In addition, the ability to access multiple points without reinsertion, as in Fig. 5, would facilitate the delivery of any desired therapy that can be infused through a needle or catheter: toxins, dendritic cells, tagged antibodies, radioactive particles, therapeutic genes, or chemotherapeutic agents could be delivered with considerable versatility, accessing a large number of points within the tumor, but using just one single main trajectory channel through normal brain tissue. The dose delivery to targeted cells would be made more precise, and runoff into surrounding normal brain would be minimized.

The results presented demonstrate qualitatively the concepts described. Future research will require comprehensive testing of the duty-cycled needle steering approach and quantitative characterization of the performance of a wider variety of needle prototypes. A suitable approach for image guidance will also be sought, to be followed by tests in animal tissue in vitro.

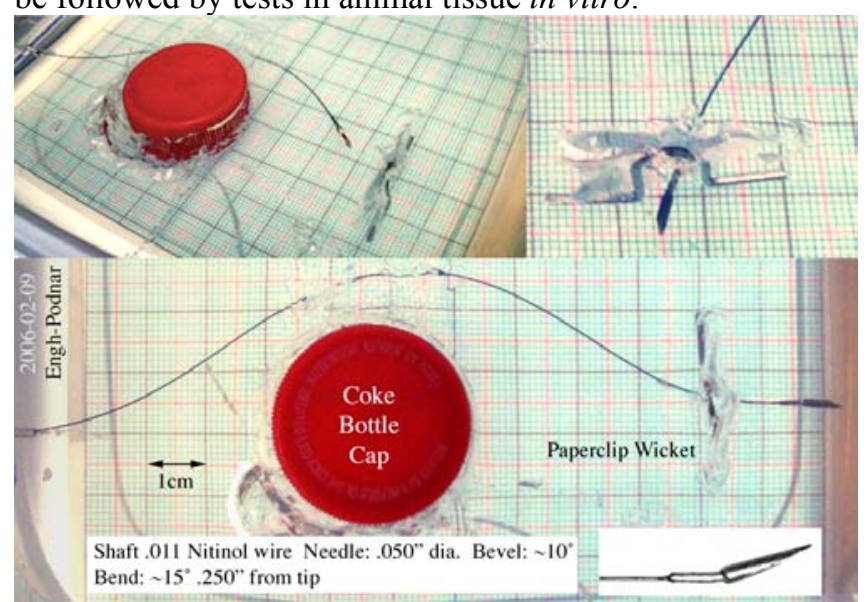

Fig. 4. Qualitative demonstration of a detour around a plastic bottle cap (representing a critical part of the brain) followed by passage through a small metallic wicket (representing a target lesion). This test was performed using the second custom needle prototype (Fig. 3(b)). The black gridlines are $1 \mathrm{~cm}$ apart.

\section{REFERENCES}

[1] S. H. Landis, T. Murray, S. Bolden and P. A. Wingo, "Cancer statistics, 1999," CA Cancer J. Clin., vol. 49, no. 1, pp. 8-31, 1999.

[2] E. M. Kemper, W. Boogerd, I. Thuis, J. H. Beijnen, O. Van Tellingen, "Modulation of the blood-brain barrier in oncology: therapeutic opportunities for the treatment of brain tumors," Cancer Treat. Rev., vol. 30, no. 5, pp. 415-423, 2004.

[3] I.F. Dunn and P.M. Black, "The neurosurgeon as local oncologist: cellular and molecular neurosurgery in malignant glioma therapy," Neurosurgery 52(6):1411-1422, 2003.

[4] H. Brem, S. Plantadosi, P.C. Burger, M. Walker, R. Selker, N.A. Vick, K. Black, M. Sisti, S. Brem, G. Mohr, R. Morawetz, S.C. Schold, "Placebo-controlled trial of safety and efficacy of intraoperative controlled delivery by biodegradable polymers of chemotherapy for recurrent gliomas," Lancet 345:1008-1012, 1995.
[5] S. Valtonen, U. Timonen, et al., "Interstitial chemotherapy with carmustine loaded polymers for high-grade gliomas: a randomized double-blind study," Neurosurgery 41:44-49, 1997.

[6] M. Westphal, D.C. Hilt, E. Bortey, P. Delavault, R. Olivares, P.C. Warnke, I.R.W. Jaaskelainen, Z. Ram, "A phase 3 trial of local chemotherapy with biodegradable carmustine (BCNU Gliadel wafers) in patients with primary malignant glioma. Neurooncology 5(2):79-88, 2003.

[7] National Institute of Neurological Disorders and Stroke (NINDS), and National Cancer Institute, "Report of the Brain Tumor Progress Review Group," NIH Publication Number 01-4902, 2000.

[8] Hall WA, Rustamzadeh E, Asher AL (2003) Convection-enhanced delivery in clinical trials. Neurosurg. Focus 14:1-4.

[9] M. Westphal and P.M. Black, "Perspectives of cellular and molecular neurosurgery," J Neurooncol 70(2):255-269, 2004.

[10] S. P. DiMaio and S. E. Salcudean, "Needle insertion modeling and simulation," IEEE Trans. Rob. Autom., vol. 19, no. 5, pp. 864-875, 2003

[11] N. Abolhassani, R. Patel, and M. Moallem, "Trajectory generation for robotic needle insertion in soft tissue," Proc. $26^{\text {th }}$ Annu. Int. Conf. IEEE Eng. Med. Biol. Soc., Sept. 2004, pp. 2730-2733.

[12] R.J. Webster III, J.S. Kim, N.J. Cowan, G.S. Chirikjian, and A.M. Okamura, "Nonholonomic modeling of needle steering," Int. J. Rob. Res., vol. 25, no. 5-6, pp. 509-525, 2006.

[13] R.J. Webster III, J. Mernisevic, and A.M. Okamura, "Design considerations for robotic needle steering," Proc. IEEE Int. Conf. Rob. Autom., pp. 3599-3605, 2005.

[14] J.A. Engh, G. Podnar, S.Y. Khoo, and C.N. Riviere, "Flexible needle steering system for percutaneous access to deep zones of the brain," Proc. 32 $2^{\text {nd }}$ IEEE Northeast Bioeng. Conf., pp. 103-104, 2006.

[15] R.C. Ritter, E.G. Quate, G.T. Gillies, M.S. Grady, M.A. Howard III, and W.M. Broaddus, "Measurement of friction on straight catheters in in vitro brain and phantom material," IEEE Trans. Biomed. Eng. 45(4):476-485, 1998.

[16] R. Alterovitz, K. Goldberg, and A. Okamura, "Planning for steerable bevel-tip needle insertion through 2D soft tissue with obstacles," Proc. IEEE Intl. Conf. Rob. Autom., Apr. 2005, pp. 1652-1657.

[17] S. Okazawa, R. Ebrahimi, J. Chuang, S. E. Salcudean, and R. Rohling, "Hand-held steerable needle device," IEEE/ASME Trans. Mechatron., vol. 10 , no. 3, pp. 285-296, 2005.

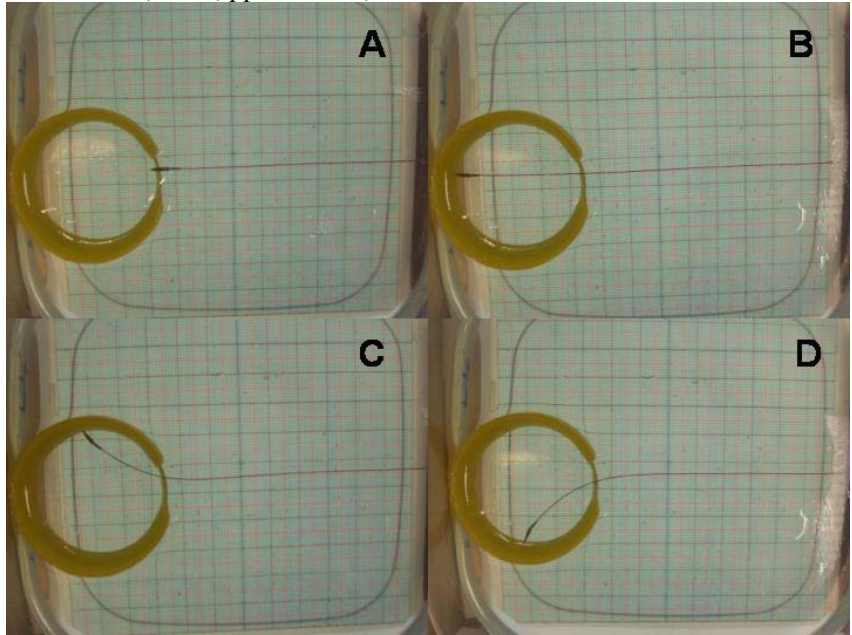

Fig. 5. Qualitative demonstration of "coverage" of different points within a lesion zone, such as might be used in local tumor therapy. The needle is steered to the edge of a treatment zone (A) (represented by a plastic bottle cap placed within the tissue substrate, with a small hole cut in its side for entry). The needle is then advanced straight forward to point (B). Then the needle returns to the entry point (A), and is advanced to other points in the treatment zone $(C$, then $D)$, each time returning to the same starting point (as in A). If the points were at the margins of a tumor, then any therapy deliverable through a catheter could be delivered to these and other points around the margin, providing coverage of the three-dimensional outline of the lesion while using only one trajectory to reach the border of the zone. This test was performed using the second custom needle prototype (Fig. $3(\mathrm{~b})$ ). The black gridlines are $1 \mathrm{~cm}$ apart. 